\title{
Long non-coding RNA C5orf66-AS1 is downregulated in pituitary null cell adenomas and is associated with their invasiveness
}

\author{
GUOQIANG YU ${ }^{1,2}$, CHUZHONG LI $^{2,3,4,5}$, WEIYAN XIE ${ }^{2}$, ZHUANG WANG ${ }^{2}$, HUA GAO $^{2}$, \\ LIHUA CAO $^{6}$, LINGTONG HAO ${ }^{6}$ and YAZHUO ZHANG ${ }^{2,3,4,5}$ \\ ${ }^{1}$ Medical Center, Tsinghua University, Haidian, Beijing 100084; ${ }^{2}$ Beijing Neurosurgical Institute, \\ Capital Medical University; ${ }^{3}$ Department of Neurosurgery, Beijing Tiantan Hospital, Capital Medical University; \\ ${ }^{4}$ Beijing Institute for Brain Disorders Brain Tumor Center; ${ }^{5}$ China National Clinical Research Center for \\ Neurological Diseases, Dongcheng, Beijing 100050; ${ }^{6}$ Genome Wisdom Inc., Haidian, Beijing 100195, P.R. China
}

Received December 15, 2016; Accepted May 22, 2017

DOI: 10.3892/or.2017.5739

\begin{abstract}
Pituitary null cell adenoma is a challenging clinical condition, and its pathogenesis remains to be elucidated. We performed this study to determine the roles of C5orf66-AS1, NORAD, and TINCR in the pathogenesis and invasion of pituitary null cell adenomas. Expression of the three long non-coding RNAs in pituitary null cell adenoma tissues of 11 patients and normal pituitary tissues from four donors was examined by performing quantitative reverse transcriptionpolymerase chain reaction. We found that C5orf66-AS1 expression was lower in pituitary null cell adenoma tissues than in normal pituitary tissues. Moreover, C5orf66-AS1 expression level was significantly lower in invasive pituitary null cell adenomas than in non-invasive ones. After transfection of C5orf66-AS1 into pituitary adenoma cells, assessment of cell viability and invasion suggested that overexpressed C5orf66-AS1 inhibited cell viability and cell invasion. In silico algorithms predicted several cis-and trans-acting target genes of C5orf66-AS1, including PITX1 and SCGB3A1. In addition, expression of some of the predicted target genes was determined using microarray data of another cohort with pituitary null cell adenomas. It showed that some of these target genes were differentially expressed between pituitary null cell adenoma tissues and normal pituitary tissues as well
\end{abstract}

Correspondence to: Professor Yazhuo Zhang, Beijing Neurosurgical Institute, 6 Tiantan Xili, Dongcheng, Beijing 100050, P.R. China

E-mail: zyz2004520@yeah.net

Abbreviations: Gd, gadolinium; GTEx, Genotype-Tissue Expression; lincRNAs, long intergenic non-coding RNAs; IncRNAs, long non-coding RNAs; MRI, magnetic resonance imaging; qRT-PCR, quantitative reverse transcription-polymerase chain reaction; RNA-seq, RNA sequencing

Key words: C5orf66-AS1, NORAD, TINCR, lincRNA, pituitary null cell adenomas as between invasive and non-invasive tumors. Co-expression analysis in RNA sequencing data showed that $P A Q R 7$ was the most correlated gene of C5orf66-AS1 and that several predicted trans-acting target genes, including $S C G B 3 A 1$, were highly correlated with C5orf66-AS1. NORAD and TINCR expression was not statistically significant in the complete cohort; however, a negative correlation was observed between NORAD expression and maximum tumor diameter in some subgroups. These results indicate that C5orf66-AS1 suppresses the development and invasion of pituitary null cell adenomas. However, our results do not provide enough statistical evidence to support the roles of NORAD and TINCR in the development and invasion of pituitary null cell adenomas.

\section{Introduction}

Pituitary adenoma is one of the most common intracranial tumors, with an increasing prevalence in recent years (1). Of its multiple subtypes, pituitary null cell adenoma was originally defined by electron microscopy as a tumor containing cells with relatively few, poorly developed organelles and with no sufficiently distinctive structural characteristics to determine its cellular origin; moreover, most cells in pituitary null cell adenomas do not yield positive immunostaining results for anterior pituitary hormones (2,3). Null cell adenomas, including their oncocytic variants, account for approximately $25.1 \%$ of all pituitary adenomas, and gonadotropinomas account for approximately $24.8 \%$ of all pituitary adenomas (3). Pituitary null cell adenomas are clinically challenging because they often present as macroadenomas and because there are no reliable biomarkers for monitoring and effective targeted therapy for treating these tumors. Moreover, the origin and pathogenesis of pituitary null cell adenomas remain to be elucidated to date.

Long non-coding RNAs (lncRNAs) are transcribed from at least $75 \%$ of the human genome (4). They play important roles in gene regulation and maintenance of genomic stability and affect various cellular process, including survival, proliferation, and migration (5). Several lncRNAs function as oncogenes, tumor suppressors, or prognostic markers (6-10). Recent studies have reported that some novel tumor-associated lncRNAs participate in the pathogenesis of different tumors, 
such as TINCR, which is underexpressed and acts as a tumor suppressor in colorectal cancer (11). C5orf66-AS1 (also known as Epist and CTC-276P9.1) is underexpressed in esophageal squamous cell carcinomas compared with that in normal esophageal tissues (12). Results of functional experiments have shown that C5orf66-AS1 functions as a tumor suppressor (12). A more recent study showed that expression of NORAD, which stabilizes the genome and whose inactivation results in dramatic aneuploidy (13), was elevated in invasive breast cancer and was associated with decreased survival, and showed that in vitro knockdown of NORAD inhibited tumor growth (14). To date, only a few studies have examined roles of lncRNAs in pituitary adenomas.

From RNA sequencing (RNA-seq) data of another cohort with various types of pituitary adenomas, which is under study in our laboratory, we carried out a manual review of the aberrantly expressed long intergenic non-coding RNAs (lincRNAs) of the null cell type. We found that the lncRNAs C5orf66-AS1, NORAD, and TINCR were in the list of the 120 most significantly dysregulated lincRNAs, and after the manual review we were specifically interested in the functions of these three lincRNAs and their targets. Since few studies have examined roles of IncRNAs in pituitary null cell adenomas, we wondered if they play similar roles in the development of pituitary null cell adenomas based on previous studies on the participation of these three lncRNAs in oncogenesis of other tumors.

\section{Materials and methods}

Examining gene expression abundance across multiple tissues online. We first examined the expression of three lncRNAs, C5orf66-AS1, NORAD, and TINCR, in different normal adult tissues by using RNA-seq data from the GenotypeTissue Expression (GTEx) Project (http://www.gtexportal.org/ home/). The data described in this study were retrieved from the GTEx Portal 11/17/2016 (GTEx Analysis Release V6p) and dbGaP (accession no. phs000424.v6.p1) 11/17/2016.

Patients and specimens. Patients who underwent endoscopic trans-sphenoidal surgery from March 2013 to November 2014 at Beijing Tiantan Hospital and who were diagnosed with pituitary null cell adenomas through immunostaining and electron microscopy were enrolled in the present study. Inclusion criteria were results of electron microscopy showing cells with relatively few, poorly developed organelles; absence of sufficiently distinctive structural characteristics to determine the cellular origin of the tumor; and negative results for the immunostaining of anterior pituitary hormones, which was consistent with the definition of pituitary null cell adenoma described above and according to the World Health Organization classification (2004 version) $(2,3,15)$. Patients with a family history of pituitary adenoma or other tumors of the endocrine system were excluded. Each patient underwent a hormonal serum test and magnetic resonance imaging (MRI) before undergoing surgery. The excised tissues were examined by performing histopathological and immunohistochemical analysis for all anterior pituitary hormones and by electron microscopy. Tumors with Hardy-Wilson classification grade IV and/or Knosp classification grades III and IV (16-18) were defined as invasive. Invasiveness was determined by a group of
Table I. Sequences of primers used in qRT-PCR.

\begin{tabular}{ll}
\hline Gene & \multicolumn{1}{c}{ Primer sequence } \\
\hline TINCR & F: AAGGAGAGCCTACTTCCCTCAA \\
& R: TCTAGTTCCAAGCTGGGTGATC \\
C5orf66-AS1 & F: GCTTCGCGTCAAGAGGGTAT \\
& R: GACCGACGTCTGCTGCTTTT \\
NORAD & F: AGCTTTGGGATTTTGAATTGGT \\
& R: GATCCTGTGTGTAGGCACAACAT \\
SCGB3A1 & F: ACAATGTTCGGTTGAGGGGAA \\
& R: AGGTGTGAGCAGCAGGGTTC \\
FAPDH & F: ACAGCCTCAAGATCATCAGCAAT \\
& R: GATGGCATGGACTGTGGTCAT \\
\hline
\end{tabular}

F, forward; R, reverse.

neurosurgeons, radiologists, and pathologists. Maximum tumor diameter was measured using gadolinium $(\mathrm{Gd})$-enhanced T1WI MR images. Normal pituitary tissues were obtained from donors who died from diseases other than neurologic or endocrine diseases. All patients provided written informed consent, and the study was approved by the Ethics Committee of the Beijing Tiantan Hospital.

Total RNA extraction and cDNA synthesis. Pituitary adenoma and normal anterior pituitary tissues were stored in liquid nitrogen immediately after removal. Total RNA was extracted from the frozen tissue samples by using TRizol (Invitrogen, Carlsbad, CA, USA), according to the manufacturer's instructions. Integrity of the total RNA was determined by performing agarose gel electrophoresis. Only samples showing no RNA degradation $(28 \mathrm{~S} / 18 \mathrm{~S} \geq 0.7)$ were used to generate labeled targets. RNA purity and concentration were determined using NAS-99 (Merinton, Beijing, China) and A260:A280 ratios yielded were consistently close to 2.0. Total RNA was reverse transcribed to cDNA by using HiFiScript gDNA Removal cDNA Synthesis kit (CWBio Co., Ltd., Beijing, China), according to the manufacturer's instructions.

Quantitative reverse transcription-polymerase chain reaction $(q R T-P C R)$. qRT-PCR was performed using StepOnePlus ${ }^{\mathrm{TM}}$ Real-Time PCR System (Applied Biosystems, Foster City, CA, USA) and Kapa SYBR FAST qPCR Master Mix ABI Prism ${ }^{\mathrm{TM}}$ (2X, KK4601; Kapa Biosystems, Inc., Wilmington, MA, USA), according to the manufacturer's instructions. SYBR Green assays were performed using $0.5 \mu \mathrm{l}$ PCR forward primer $(10 \mu \mathrm{M}), 0.5 \mu \mathrm{l}$ PCR reverse primer $(10 \mu \mathrm{M}), 2 \mu \mathrm{l}$ cDNA, $5 \mu \mathrm{l}$ $2 \mathrm{X}$ master mix, and $2 \mu \mathrm{l}$ double distilled water in a total reaction volume of $10 \mu 1$. The PCR protocol was initiated at $95^{\circ} \mathrm{C}$ for $3 \mathrm{~min}$, followed by 40 cycles of $95^{\circ} \mathrm{C}$ for $10 \mathrm{sec}$ and $60^{\circ} \mathrm{C}$ for $60 \mathrm{sec}$. GAPDH was used as a reference gene. Results were obtained using three independent wells. Relative expression level was calculated using $2^{-\Delta \Delta \mathrm{Ct}}$ method (19) after normalizing with $G A P D H$ expression level. Sequences of primers used for performing qRT-PCR are presented in Table I. 


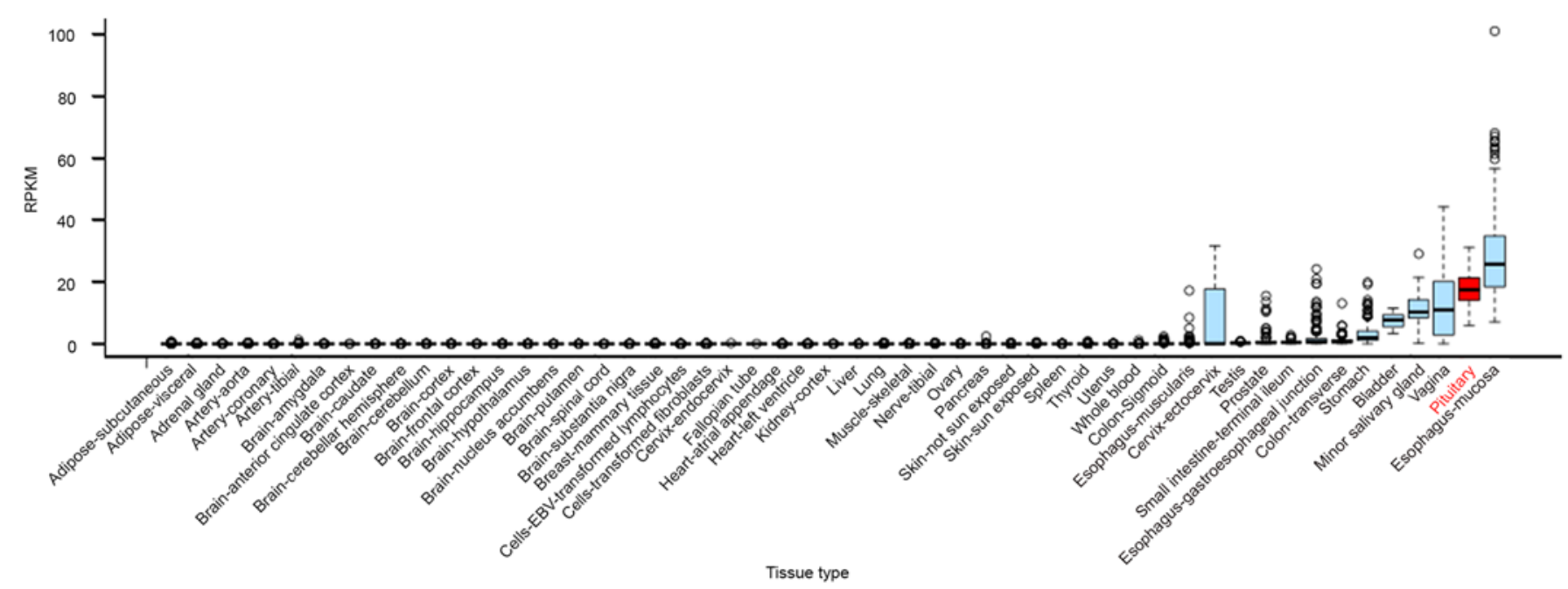

Figure 1. RNA-seq data from 53 normal human tissues showing that C5orf66-AS1 is overexpressed in the pituitary gland. These data were retrieved from GTEx (GTEx Analysis Release V6p, dbGaP accession no. phs000424.v6.p1).

In silico target gene prediction. Two algorithms were used to predict possible $\mathrm{cis}$ - and trans-acting target genes, as previously described (20). Cis-acting target genes were predicted using UCSC genome browser and genome annotation to locate the nearest known protein-coding genes from the lncRNAs (21). According to distance stratification, proteincoding genes located within a distance of 5 and $300 \mathrm{~kb}$ from the lncRNAs were captured as targets. Trans-acting genes were predicted using RNAplex software (http://www.bioinf. uni-leipzig.de/Software/RNAplex/). RNAplex can determine possible hybridization sites for a query RNA according to sequence complementarity and RNA duplex-binding energy prediction $(22,23)$. First, BLAST $(\mathrm{e}<1 \mathrm{E}-5)$ was performed using the lncRNAs and known protein-coding genes; next, RNAplex (parameter set as -e -70) was used to screen transacting target genes.

Gene expression microarray analysis. Expression of predicted target genes was determined using microarray data of another cohort examined in our laboratory. Microarray analysis was performed using Whole Human Genome Oligo Microarray (4x44K) (Agilent Technologies, Santa Clara, CA, USA). Total RNA was extracted and was purified using mirVana ${ }^{\mathrm{TM}}$ miRNA Isolation kit (catalog no. AM1561; Ambion, Austin, TX, USA), according to the manufacturer's instructions. RIN was determined using Bioanalyzer 2100 (Agilent Technologies) to inspect RNA integration. None of the samples showed RNA degradation ( $\mathrm{RIN} \geq 7.0$ and $28 \mathrm{~S} / 18 \mathrm{~S} \geq 0.7$ ). Total RNA was amplified and was labeled using Low Input Quick Amp labeling kit, One-Color (catalog no. 5190-2305; Agilent Technologies). Labeled cRNA was purified using RNeasy mini kit (catalog no. 74106; Qiagen, GmBH, Hilden, Germany). Next, each slide was hybridized with Cy3-labeled cRNA by using Gene Expression Hybridization kit (catalog no. 5188-5242; Agilent Technologies), according to the manufacturer's instructions. After hybridization, the slides were scanned using a microarray scanner (catalog no. G2565CA; Agilent Technologies). Data were extracted using Feature Extraction software 10.7 (Agilent Technologies), and raw data were normalized using Quantile algorithm, limma packages in R.
RNA sequencing. We previously performed RNA sequencing of a cohort with various types of pituitary adenomas, which is under study in our laboratory. After the total RNA extraction and DNase I treatment, magnetic beads with Oligo (dT) were used to isolate mRNAs. mRNAs were fragmented into short fragments by mixing with fragmentation buffer. Then, cDNAs were synthesized using the mRNA fragments as templates. Fragments were purified and resolved with EB buffer for end reparation and single nucleotide adenine addition and were connected with adapters. After agarose gel electrophoresis, the suitable fragments were selected for PCR amplification as templates. During the quality control steps, the Agilent 2100 Bioanalyzer (Agilent Technologies, Palo Alto, CA, USA) and ABI StepOnePlus Real-time PCR System (Applied Biosystems) were used in quantification and qualification of the sample library. Finally, the library was sequenced using the Illumina HiSeq 2000 (Illumina, San Diego, CA, USA) at the BGI-Tech Bioinformatics Institute (Shenzhen, China).

Cell culture and transfection. The mouse pituitary cell line GT1-1 was purchased from the China Infrastructure of Cell Line Resources and cultured in phenol red-free Dulbecco's modified Eagle's medium (DMEM; Invitrogen) supplemented with $10 \%$ fetal bovine serum (Gibco, Auckland, New Zealand) in a humidified incubator at $37^{\circ} \mathrm{C}$ with $5 \% \mathrm{CO}_{2}$. pUC57C5orf66-AS1 was obtained from BGI (Shenzhen, China). Transfection was performed using Lipofectamine 3000 (Invitrogen) according to the manufacturer's instructions. After $24 \mathrm{~h}, \mathrm{C} 5$ orf66-AS1 was examined using qRT-PCR in triplicates.

Assessment of cell viability. Log-phase GT1-1 cells were harvested and confirmed to be $>99 \%$ viable by trypan blue exclusion and adjusted to a density of $1 \times 10^{5}$ cells $/ \mathrm{ml}$. A volume of $100 \mu \mathrm{l}$ of cell suspension was plated into 96-well plates and cultured for $24 \mathrm{~h}$. After transfection for 24, 48, and $72 \mathrm{~h}, 20 \mu \mathrm{l}$ MTS solution was added to each well and further incubated for $4 \mathrm{~h}$. Absorbance of each well at $490 \mathrm{~nm}$ was measured using an ELISA plate reader (Thermo Labsystems, Franklin, MA, USA). 


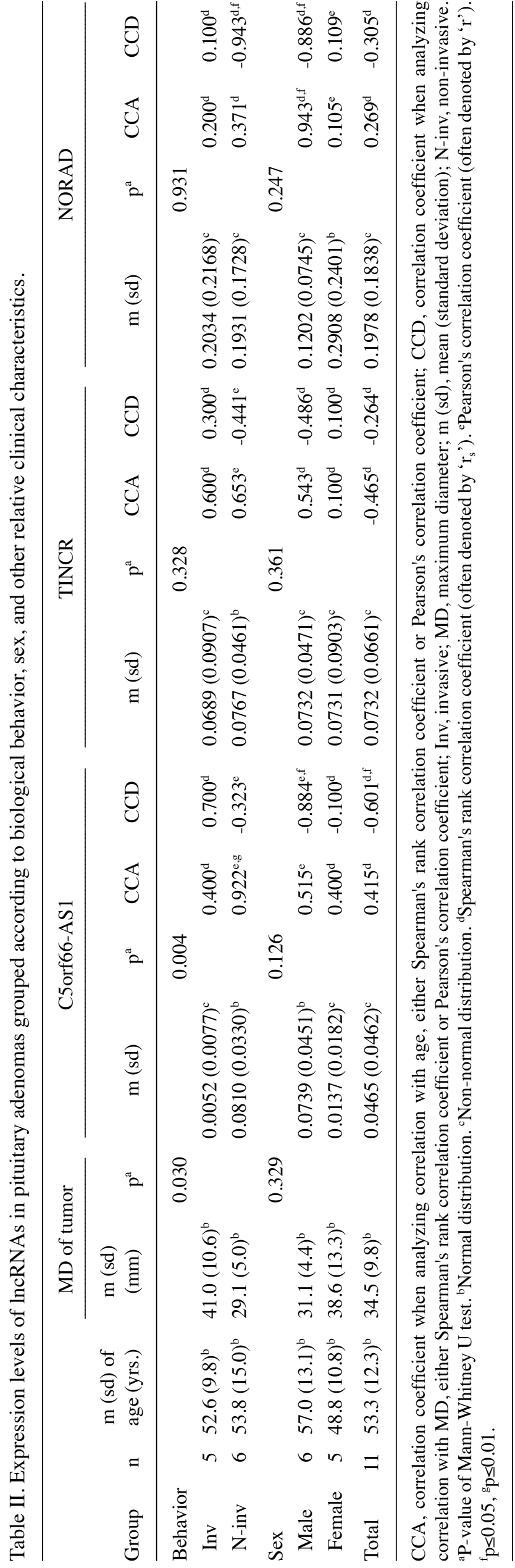

Transwell invasion assay. Tumor cell invasion was measured by examining cell migration and invasion through fibronectinand Matrigel-coated polycarbonate filters, respectively, using modified Transwell chambers (Corning Inc., Corning, NY, USA). GT1- 1 cells $\left(2 \times 10^{4}\right.$ cells $)$ were added into the upper chambers. Migrated cells adhered to the lower membrane were fixed in $4 \%$ paraformaldehyde and stained with hematoxylin (Zhongshan Company, Beijing, China). The average number of migrated cells was quantified by counting five random highpower fields under a fluorescent microscope (Carl Zeiss, Jena, Germany). Experiments were performed in triplicates.

Statistical analyses. Statistical analysis of qRT-PCR results was performed using SPSS software (v23.0; IBM Corp., Armonk, NY, USA). Shapiro-Wilk test was used to test normality. Differences between the groups were determined using Mann-Whitney U test. Correlation between IncRNA expression level and maximum tumor diameter and between lncRNA expression level and patient age was determined using Spearman's rank correlation coefficient or Pearson's correlation coefficient on the basis of data distribution. Relationship between C5orf66-AS1 expression level and maximum tumor diameter in male patients was determined by performing simple linear regression analysis after calculating Pearson's correlation coefficient. Co-expression analysis was performed with $\mathrm{R}$ based on the Pearson correlation coefficients between C5orf66-AS1 and all mRNAs with unique mapping from RNA-seq data after ruling out types of pituitary adenomas other than the null cell type. $\mathrm{P} \leq 0.05$ was considered statistically significant, except in the Shapiro-Wilk test, in which 0.1 was used as the level of significance to decrease the probability of false-negative errors.

\section{Results}

Expression of the three IncRNAs across multiple tissues in GTEx. First, we examined the expression of three IncRNAs, C5orf66-AS1, NORAD, and TINCR, in different normal tissues by using RNA-seq data from GTEx. We found that C5orf66-AS1 was overexpressed specifically in the pituitary gland, with its expression level being only lower than that in esophageal mucosa among the 53 tissue types examined (Fig. 1). TINCR was expressed at an average level, and no data are available on the expression of NORAD. These results suggest that C5orf66-AS1 performs special functions in the pituitary gland.

Clinical characteristics of patients. qRT-PCR analysis of TINCR, C5orf66-AS1, and NORAD was performed using normal pituitary tissues from four donors and tumor tissues from 11 patients, including six men and five women. Characteristics of the patients are shown in Table II. Ages of the patients ranged from 36 to 74 years, with an average age of 53.3 years. The main complaints of the patients were headache and visual disturbances; in some patients, pituitary null cell adenoma was diagnosed by performing incident MRI or CT scan. Time interval from the appearance of the first symptoms to surgery varied from 20 days to 9 years. For each patient, serum test yielded negative results for excessive pituitary hormone secretion, the sella turcica showed 


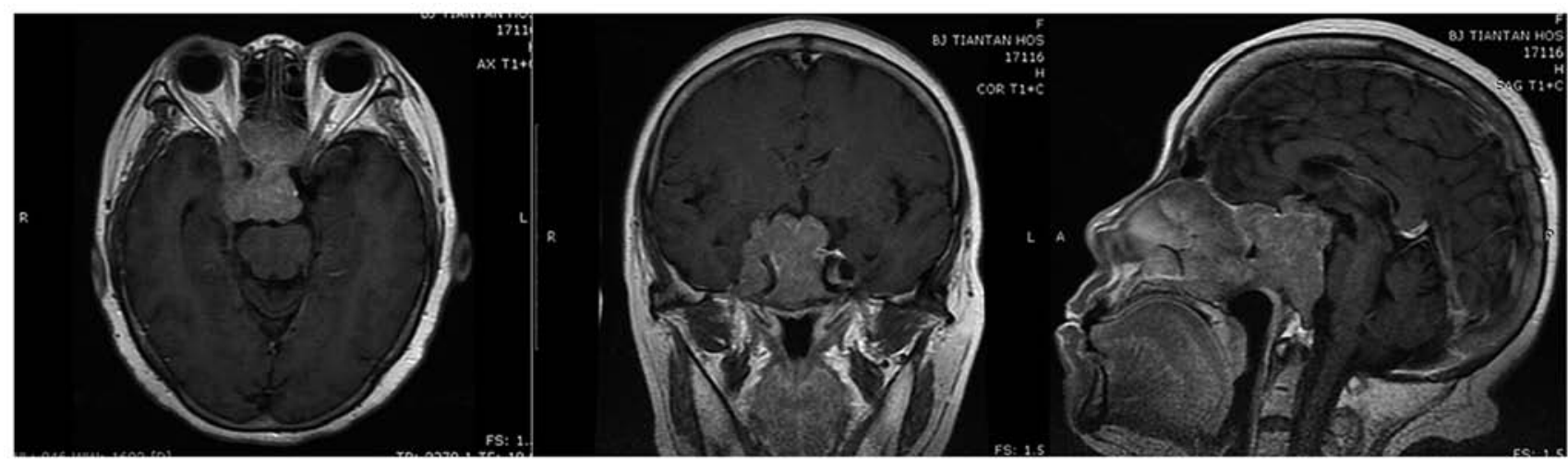

Figure 2. MR images (Gd-enhanced T1WI) of a patient with Hardy-Wilson classification grade IV and Knosp classification grade IV tumors.
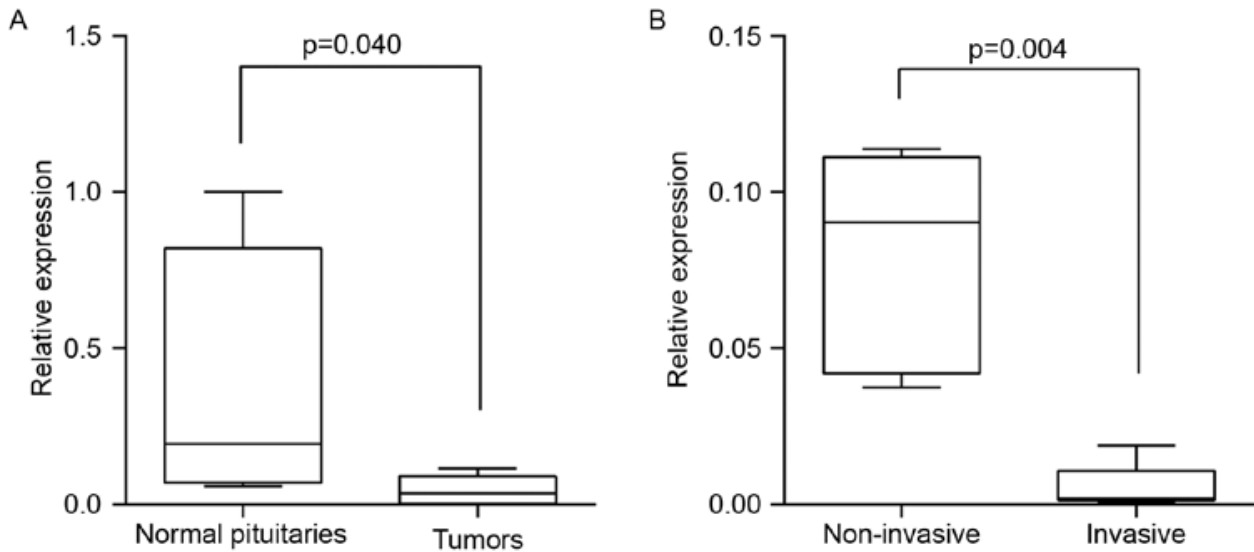

Figure 3. C5orf66-AS1 expression level is higher in normal pituitary tissues than in pituitary adenoma tissues (A) and in non-invasive pituitary adenoma tissues than in invasive pituitary adenoma tissues (B). The boxes extend from the 25th to 75th percentile with lines in the middle of boxes indicating the median, and the whiskers go down to the smallest value and up to the largest.

enlargement, and radiologic performance was consistent with the diagnosis of pituitary adenoma. Tumors of six patients were invasive; of these, three patients had HardyWilson grade III and Knosp classification grade III tumors and the remaining three patients had Hardy-Wilson grade IV and Knosp classification grade IV tumors (Fig. 2). Maximum tumor diameters ranged from 23.0 to $57.2 \mathrm{~mm}$. No differences were observed in maximum tumor diameters between male and female patients. All the tumors were diagnosed as pituitary null cell adenomas, with no major difficulty; some of these tumors showed oncocytic changes.

After predicting target genes, we examined some target genes by using microarray data obtained from pituitary null cell adenomas of 16 patients and normal pituitary tissues of six donors from another study cohort. Patients in this cohort were admitted during the same period and were diagnosed using the same criteria as those described above. Of the 16 patients, 12 were men and 4 were women. Moreover, six patients had invasive tumors, and nine patients had noninvasive tumors (invasiveness of the tumor of one patient could not be evaluated because of the absence of MRI data). Other clinical characteristics of these patients were similar to those of the 11 patients included in the present study. Then, we performed a co-expression analysis of the RNA-seq data of another cohort, including 12 pituitary null cell adenomas with other types of pituitary adenomas and five normal pituitaries. Of the 12 patients of pituitary null cell adenomas, six were men and six were women; seven patients had invasive tumors, and 5 patients had non-invasive tumors. Other clinical characteristics of these patients were similar to those of the 11 patients included in the present study.

Expression of C5orf66-AS1. C5orf66-AS1 expression level was significantly lower in pituitary null cell adenoma tissues than in normal pituitary tissues (Mann-Whitney U test, $\mathrm{Z}=-2.089$; two-tailed, $\mathrm{P}=0.040$; Table II, Fig. 3A). Moreover, C5orf66-AS1 expression level was significantly lower in invasive tumors than in noninvasive tumors (Mann-Whitney $\mathrm{U}$ test, $\mathrm{Z}=-2.739$; two-tailed, $\mathrm{P}=0.004$; Table II, Fig. 3B).

No correlation was observed between C5orf66-AS1 expression level and patient age. Analysis of the relationship between C5orf66-AS1 expression and pituitary tumor growth showed that C5orf66-AS1 lncRNA levels were negatively correlated with maximum tumor diameter (Spearman's rank correlation coefficient $r_{s}=-0.601, P=0.05$; Table II, Fig. 4A). Stratification of tumors based on sex showed a negative linear correlation between C5orf66-AS1 lncRNA levels and maximum tumor diameter in male patients (Pearson correlation: $r=-0.884$, $\mathrm{P}=0.019$; simple linear regression analysis: $\mathrm{R}^{2}=0.782$, adjusted $\mathrm{R}^{2}=0.728, \mathrm{~F}=14.360, \mathrm{P}=0.019, \mathrm{Y}=0.354-0.009 \mathrm{X}, \mathrm{t}=-3.790$, 

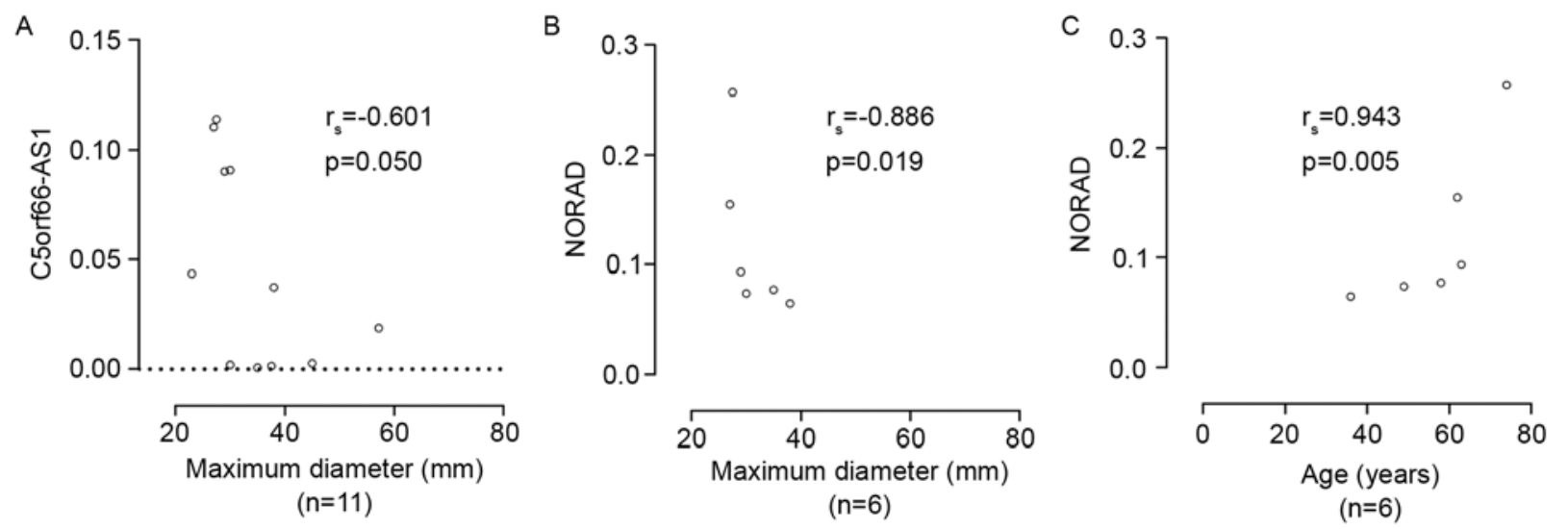

Figure 4. Relative expression of C5orf66-AS1 (A) and NORAD (B and C) is shown using scatter plots obtained from all the patients (A) and male patients (B and C). (A) A negative correlation between C5orf66-AS1 expression level and maximum tumor diameter (Spearman's rank correlation). (B) A negative correlation between NORAD expression level and maximum tumor diameter (Spearman's rank correlation). (C) A positive correlation between NORAD expression level and patient age (Spearman's rank correlation).

Table III. Cis-acting and top 20 trans-acting target genes of C5orf66-AS1 predicted in silico.

\begin{tabular}{|c|c|}
\hline Type & Gene name \\
\hline$C i s^{\mathrm{a}}$ & C5orf66, PITX1 ${ }^{\mathrm{d}}$, CATSPER3, PCBD2, TXNDC15 ${ }^{\mathrm{d}}, \mathrm{C} 5$ orf24 ${ }^{\mathrm{d}}, \mathrm{DDX} 46, \mathrm{CAMLG}, \mathrm{H} 2 \mathrm{AFY}$ \\
\hline $\operatorname{Trans}^{\mathrm{b}}$ & 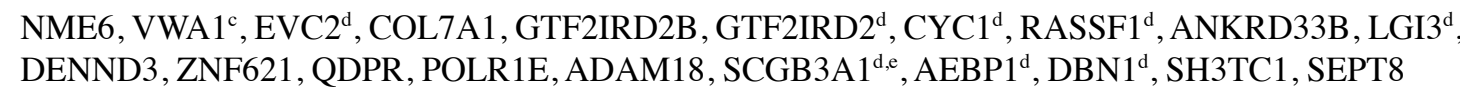 \\
\hline
\end{tabular}

$\mathrm{P}=0.019$; Table II). However, this linear correlation was not observed in female patients. Moreover, no significant difference was observed in C5orf66-AS1 expression between male and female patients. Stratification of tumors based on their invasive potential showed a significant positive linear correlation between C5orf66-AS1 expression and patient age in patients with non-invasive tumors (Pearson correlation, $\mathrm{r}=0.922, \mathrm{P}=0.009$; Table II). However, no correlation was observed between maximum tumor diameter and patient age; and this linear correlation was not observed in patients with invasive tumors.

Expression of NORAD and TINCR. NORAD and TINCR expression did not show normal distribution in the tumor samples of the complete cohort examined in the study. No difference was observed in NORAD and TINCR expression levels between tumor and normal pituitary tissues and between invasive and non-invasive tumors. Moreover, no correlation was observed between NORAD and TINCR expression levels and maximum tumor diameter or patient age. However, in non-invasive pituitary null cell tumors, NORAD lncRNA level was significantly negatively correlated with maximum tumor diameter (Spearman's rank correlation, $r_{s}=-0.943$, $\mathrm{P}=0.005$; Table II). Furthermore, NORAD IncRNA level was significantly negatively correlated with maximum tumor diameter (Spearman's rank correlation, $\mathrm{r}_{\mathrm{s}}=-0.886, \mathrm{P}=0.019$;
Table II, Fig. 4B) but significantly positively correlated with age (Spearman's rank correlation, $\mathrm{r}_{\mathrm{s}}=0.943, \mathrm{P}=0.005$; Table II, Fig. 4C) in male patients. However, no correlation was observed between maximum tumor diameter and age in male patients.

In silico prediction of target genes of C5orf66-AS1 and their expression in microarrays. Nine cis-acting target genes of C5orf66-AS1 were detected in a distance of $<300 \mathrm{~kb}$ from C5orf66-AS1 (Table III). Of these, the top two genes, namely, C5orf66 and PITX1, were within a distance of $5 \mathrm{~kb}$ from C5orf66-AS1. Location of C5orf66-AS1 is in chromosome 5: 135,038,831-135,040,047 (reverse strand), while that of C5orf66 is in chromosome 5: 135,033,280-135,344,680 (forward strand) and PITX1 is chromosome 5: 135,027,734-135,034,274 (reverse strand) (in assembly of GRCh38.p7/GCF_000001405.33, Fig. 5). Furthermore, we predicted 347 trans-acting target genes of C5orf66-AS1. The top 20 trans-acting genes with the best interaction energy are listed in Table III.

Next, we examined the predicted target genes (nine cis-acting genes and top 20 trans-acting genes) in microarray data of another study cohort (16 patients with pituitary null cell adenomas and six donors with normal pituitary gland) examined in our laboratory. By using a stringency of $\mathrm{P} \leq 0.01$ and fold change of $>1.5$, we found that 3 of the 9 predicted cis-acting genes and 8 of the 20 predicted trans-acting genes were differentially expressed between tumor tissues and 


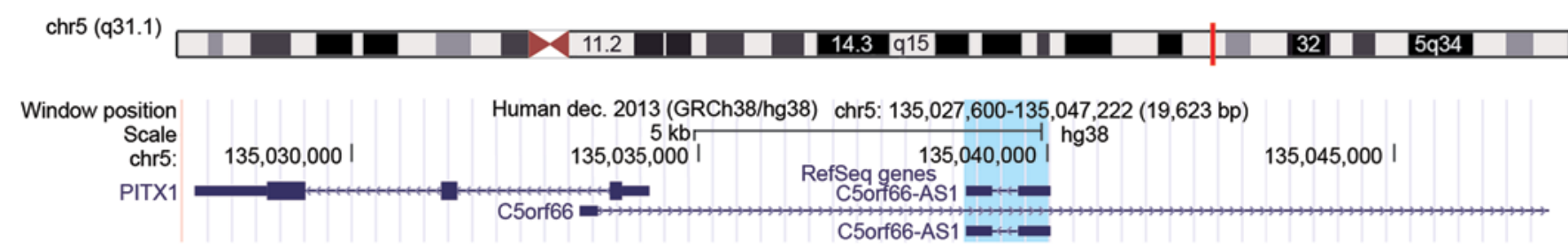

Figure 5. Location of C5orf66-AS1, C5orf66, and PITX1 (GRCh38.p7/GCF_000001405.33).

normal pituitary tissues (Table III). Moreover, by using a stringency of $\mathrm{P} \leq 0.05$ and fold change of $>1.5$, we found that only $S C G B 3 A 1$ was significantly differentially expressed in invasive and non-invasive pituitary null cell adenomas $(\mathrm{P}=0.046$, fold change $=6.817)$. Then, we performed qRT-PCR to validate expression of $S C G B 3 A 1$ in pituitary null cell adenomas and in normal pituitary tissues. The result showed that $S C G B 3 A 1$ was significantly underexpressed in pituitary adenomas compared with normal pituitaries $(\mathrm{P}=0.030)$ and was significantly underexpressed in invasive tumors compared with non-invasive ones $(\mathrm{P}=0.041)$.

Co-expression analysis of C5orf66-AS1 in RNA-seq data. Co-expression analysis of C5orf66-AS1 in the RNA-seq data showed that among the total 19,378 mRNAs which were uniquely mapped to, there were 32 mRNAs with $r>0.90$ or $\mathrm{r}<-0.90(\mathrm{P}<0.001)$ and 3,002 mRNAs with $\mathrm{r}>0.70$ or $<-0.70$ $(\mathrm{P}<0.001)$. The most correlated gene was PAQR7 $(\mathrm{r}=0.938$, $\mathrm{P}<0.001)$. In the top 20 trans-acting genes we predicted in silico, there were eight genes with $\mathrm{r}>0.70(\mathrm{P}<0.001)$. They were NME6 $(\mathrm{r}=0.851)$, RASSF1 $(\mathrm{r}=0.822)$, EVC2 $(\mathrm{r}=0.821)$, COL7A1 (r=0.746), POLRIE (r=0.730), SCGB3A1 ( $\mathrm{r}=0.730)$, LGI3 (r=0.728), and GTF2IRD2 ( $\mathrm{r}=0.723)$. Out of these eight genes, seven genes (NME6, RASSF1, EVC2, COL7A1, $S C G B 3 A 1, L G I 3$, and GTF2IRD2) were differentially expressed between tumor tissues and normal pituitary tissues.

Cell viability and invasion assay after overexpressing C5orf66-AS1. To confirm the tumor suppressor role of C5orf66-AS1, we first overexpressed it in mouse pituitary cell line GT1-1 by transfection. We performed qRT-PCR to examine expression of C5orf66-AS1 before transfection and at $24 \mathrm{~h}$ after transfection. The results showed that C5orf66-AS1 was significantly overexpressed after transfection. A cell viability assay was performed at 24,48 , and $72 \mathrm{~h}$ after transfection. The result showed that after C5orf66-AS1 transfection, the viability of tumor cells was significantly inhibited compared with that of the control group in a time-dependent manner. After that, we performed the Transwell invasion assay. The result showed that overexpression of C5orf66-AS1 markedly reduced the effect of invasion.

\section{Discussion}

Null cell adenoma is a special type of pituitary adenoma, which has no structural characteristics sufficient to determine its cellular origin and which does not yield positive immunostaining results for adenohypophyseal hormones, except for a few scattered cells (2). Results of electron microscopy show that pituitary null cell adenoma cells have special features such as electron-lucent cytoplasm, with relatively few, poorly developed organelles and small, spherical, and usually sparse secretory granules (2,3). Although pituitary null cell adenomas are presumed to originate from gonadotropic cells $(3,24)$, some studies argue that null cell adenomas are different from gonadotropic adenomas $(25,26)$. However, the actual origin and detailed mechanism underlying the pathogenesis of pituitary null cell adenoma have not been elucidated to date.

From RNA-seq data of another cohort with various types of pituitary adenomas, we found that lncRNAs C5orf66-AS1, NORAD, and TINCR were significantly dysregulated in the null cell type. Since recent studies indicate that lncRNAs C5orf66-AS1 (12), NORAD $(13,14)$, and TINCR (11) are involved in the pathogenesis of other tumors, we examined whether these lncRNAs played similar roles in pituitary adenomas. We performed qRT-PCR analysis of the three lncRNAs. We found that C5orf66-AS1 expression was significantly lower in pituitary null cell adenoma tissues than in normal pituitary tissues and in invasive adenomas than in non-invasive adenomas. Moreover, we observed a negative correlation between C5orf66-AS1 expression and maximum tumor diameter in the complete cohort as well as in male patients (this was not observed in female patients possibly because of small sample size). After transfection into GT1-1 cells, assessment of cell viability and invasion suggested that overexpressed C5orf66-AS1 inhibited cell viability and cell invasion. C5orf66-AS1 acts as a tumor suppressor in different tumor types $(12,27)$. The pattern of C5orf66-AS1 expression in pituitary null cell adenomas and results of in vitro experiments were consistent with its role as a suppressor of tumor development and invasion. A significant positive linear correlation was observed between C5orf66-AS1 expression and age in patients with non-invasive pituitary null cell adenomas. This finding might be of interest; however, further studies must be conducted to validate this finding because of the small sample size used in the present study.

Next, we performed in silico prediction of target genes of C5orf66-AS1. We found that two cis-acting target genes, including PITX1 (28-32), which is important for pituitary development and tumorigenesis, were located in a distance of $<5 \mathrm{~kb}$ from C5orf66-AS1. In addition, we determined 347 trans-acting target genes by setting RNAplex parameter as -e -70. Among the top 20 trans-acting target genes with the most stable interaction (Table III), several genes such as $R A S S F 1, S C G B 3 A 1$, and $A E B P 1$ were found to be associated with transcription regulation or tumor development (33-38). Especially RASSF 1 was indicated to play a role in pituitary 
tumorigenesis and suppression of tumor progression $(33,39)$. Next, we explored the 29 target genes in microarray data obtained from another study cohort. By using a stringency of $\mathrm{P} \leq 0.01$, we found that three of the nine predicted cis-acting target genes and eight of the 20 predicted trans-acting target genes, including PITX1, RASSF1, SCGB3A1, and AEBP1, were significantly differentially expressed between tumor and normal pituitary tissues.

Notably, a considerable proportion of trans-targeting genes (8 of 20) were differentially expressed in pituitary tumor tissues compared to normal pituitary tissues; this implicates the role of a trans-acting mechanism in the effect exerted by C5orf66-AS1. Furthermore, by using a stringency of $\mathrm{P} \leq 0.05$, we found that $S C G B 3 A 1$ was differentially expressed between invasive and non-invasive pituitary null cell adenomas, which was then confirmed by qRT-PCR. Since $S C G B 3 A 1$ was the only gene that was differentially expressed in both comparisons, which was consistent with the expression pattern of C5orf66-AS1, it may be the most probable target gene of C5orf66-AS1. Next, we performed co-expression analysis of C5orf66-AS1 in the RNA-seq data. We found that the most correlated gene with C5orf66-AS1 was $P A Q R 7(\mathrm{r}=0.938)$, a membrane progesterone receptor that may mediate a reduction in $\mathrm{GnRH}$ in the progesterone negative feedback action in a PR (A/B)-independent way (40). This is interesting because pituitary is pivotal in the hypothalamic-pituitary-gonadal axis for progesterone feedback action, and several researchers have presumed that pituitary null cell adenomas originate from gonadotropic cells $(3,24)$. In the top 20 trans-acting genes we predicted in silico, there were eight genes with $r>0.70$, and seven genes out of those are differentially expressed in tumor tissues and normal pituitary tissues, including RASSF1 and SCGB3A1.

As showed in Fig. 5, C5orf66-AS1 is located within C5orf66 and is $<5 \mathrm{~kb}$ upstream of PITX1. It is transcribed in the antisense direction of $C 5$ orf66 and in the same direction as PITX1. According to the definitions provided by Luo et al (7), for PITX1, C5orf66-AS1 belongs to the biotype of sense IncRNAs, which are transcribed in the same direction as the nearby protein-coding gene and are included in the class of genic lncRNAs. Luo et al found that lncRNAs showed nonrandom genomic distribution within a $5-\mathrm{kb}$ distance from neighboring coding gene, which defines genic lncRNAs. The authors provided multiple solid proofs to identify the genuine cis-regulation of some divergent genic lncRNAs, including binding to and acting on chromatin and interacting with mediators. Their results suggest that the sense biotype of genic lncRNAs may regulate neighboring protein-coding genes in cis as well as by adjusting transcription or through another yet unknown mechanism. This function was partly validated by Wei et al (12) who determined the suppressive role of C5orf66-AS1 in esophageal squamous cell carcinoma. They determined high coexpression of three genes, namely, C5orf66-AS1, neighboring PITX1, and RASAL1, which is a major target of PITX1 (29). Knockdown of C5orf66-AS1 significantly altered the expression of two downstream targets of PITX1, namely RASAL1 and TERT. Since PITX1 is a pan-pituitary and pituitary-specific regulator of transcription involved in the early development and differentiation of the pituitary gland (28) and because it suppresses the tumorigenicity in multiple tumors (29) especially prolactinomas (32), we hypothesized that C5orf66-AS1 exerted a tumor suppressor effect partly through PITX1. However, further studies are needed to confirm this hypothesis.

Although NORAD and TINCR showed non-normal distribution most probably because of the small sample size, we obtained a few interesting results after analyzing the data further. Stratification of tumors according to their invasive potential showed that NORAD expression level was significantly negatively correlated with maximum tumor diameter in patients with non-invasive pituitary null cell adenomas. Stratification of tumors according to sex showed that NORAD expression level was significantly negatively correlated with maximum tumor diameter and was significantly positively correlated with patient age in male patients. Although these results should be validated by performing further studies involving a large sample size, the negative correlation between NORAD expression level and tumor size in the male and non-invasive subgroups suggests a tumor suppressive role of NORAD in pituitary null cell adenomas.

In summary, we found that C5orf66-AS1 expression was lower in null cell adenomas than in normal pituitary tissues and in invasive adenomas than in non-invasive adenomas. These results along with the negative correlation between C5orf66-AS1 expression and maximum tumor diameter and the results of in vitro experiments on pituitary adenoma cells indicate that C5orf66-AS1 suppresses the development and invasion of pituitary null cell adenomas. However, sufficient statistical evidence is not available to support the roles of NORAD and TINCR in the development and invasion of pituitary null cell adenomas, although our results suggest that NORAD may also play a tumor suppressive role. To our knowledge, the present study is the first to investigate the roles of these three IncRNAs in pituitary adenomas. However, further studies are needed to validate the findings of the present study and to determine detailed mechanisms underlying the functions of these lncRNAs.

\section{Acknowledgements}

We used some data from the GTEx Project, which was supported by the Common Fund of the Office of the Director of the National Institutes of Health, NCI, NHGRI, NHLBI, NIDA, NIMH, and NINDS. This study was supported by the Research Special Fund for Public Welfare Industry of Health (201402008); the National High Technology Research and Development Program of China (863 Program, 2015AA020504). An English Language Service from Elsevier's WebShop was used for language editing.

\section{References}

1. Aflorei ED and Korbonits M: Epidemiology and etiopathogenesis of pituitary adenomas. J Neurooncol 117: 379-394, 2014.

2. Kovacs K, Horvath E, Ryan N and Ezrin C: Null cell adenoma of the human pituitary. Virchows Arch A Pathol Anat Histol 387: 165-174, 1980.

3. Saeger W, Lüdecke DK, Buchfelder M, Fahlbusch R, Quabbe HJ and Petersenn S: Pathohistological classification of pituitary tumors: 10 years of experience with the German Pituitary Tumor Registry. Eur J Endocrinol 156: 203-216, 2007.

4. Djebali S, Davis CA, Merkel A, Dobin A,Lassmann T, Mortazavi A Tanzer A, Lagarde J, Lin W, Schlesinger F, et al: Landscape of transcription in human cells. Nature 489: 101-108, 2012. 
5. Huarte M: The emerging role of lncRNAs in cancer. Nat Med 21: $1253-1261,2015$

6. Zheng GXY, Do BT, Webster DE, Khavari PA and Chang HY: Dicer-microRNA-Myc circuit promotes transcription of hundreds of long noncoding RNAs. Nat Struct Mol Biol 21: 585-590, 2014

7. Luo S, Lu JY, Liu L, Yin Y, Chen C, Han X, Wu B, Xu R, Liu W, Yan P, et al: Divergent lncRNAs regulate gene expression and lineage differentiation in pluripotent cells. Cell Stem Cell 18: 637-652, 2016.

8. Li L, Liu B, Wapinski OL, Tsai MC, Qu K, Zhang J, Carlson JC, Lin M, Fang F, Gupta RA, et al: Targeted disruption of Hotair leads to homeotic transformation and gene derepression. Cell Rep 5: 3-12, 2013.

9. Chunharojrith P, Nakayama Y, Jiang X, Kery RE, Ma J, De La Hoz Ulloa CS, Zhang X, Zhou Y and Klibanski A: Tumor suppression by MEG3 lncRNA in a human pituitary tumor derived cell line. Mol Cell Endocrinol 416: 27-35, 2015.

10. Li Z, Li C, Liu C, Yu S and Zhang Y: Expression of the long non-coding RNAs MEG3, HOTAIR, and MALAT-1 in nonfunctioning pituitary adenomas and their relationship to tumor behavior. Pituitary 18: 42-47, 2015.

11. Zhang ZY, Lu YX, Zhang ZY, Chang YY, Zheng L, Yuan L, Zhang F, Hu YH, Zhang WJ and Li XN: Loss of TINCR expression promotes proliferation, metastasis through activating EpCAM cleavage in colorectal cancer. Oncotarget 7: 22639-22649, 2016.

12. Wei G, Luo H, Sun Y, Li J, Tian L, Liu W, Liu L, Luo J, He J and Chen R: Transcriptome profiling of esophageal squamous cell carcinoma reveals a long noncoding RNA acting as a tumor suppressor. Oncotarget 6: 17065-17080, 2015

13. Lee S, Kopp F, Chang T-C, Sataluri A, Chen B, Sivakumar S, Yu H, Xie Y and Mendell JT: Noncoding RNA NORAD regulates genomic stability by sequestering PUMILIO proteins. Cell 164: 69-80, 2016

14. Liu H, Li J, Koirala P, Ding X, Chen B, Wang Y, Wang Z, Wang C, Zhang $\mathrm{X}$ and Mo YY: Long non-coding RNAs as prognostic markers in human breast cancer. Oncotarget 7: 20584-20596, 2016.

15. Lloyd R, Kovacs K, Young WJ, et al: Tumour of the pituitary gland. In: World Health Organization Classification of Tumours: Pathology and Genetics of Tumours of Endocrine Organs. Delellis RA, Lloyd RV, Heitz PU and Eng C (eds). International Agency for Research and Cancer (IARC) Press, Lyon, pp9-48, 2004

16. Wilson CB: A decade of pituitary microsurgery. The Herbert Olivecrona lecture. J Neurosurg 61: 814-833, 1984

17. Knosp E, Steiner E, Kitz K and Matula C: Pituitary adenomas with invasion of the cavernous sinus space: A magnetic resonance imaging classification compared with surgical findings. Neurosurgery 33: 610-617, discussion 617-618, 1993.

18. Micko ASG, Wöhrer A, Wolfsberger S and Knosp E: Invasion of the cavernous sinus space in pituitary adenomas: Endoscopic verification and its correlation with an MRI-based classification J Neurosurg 122: 803-811, 2015.

19. Livak KJ and Schmittgen TD: Analysis of relative gene expression data using real-time quantitative PCR and the 2(-Delta Delta C(T)) method. Methods 25: 402-408, 2001.

20. Lin XC, Zhu Y, Chen WB, Lin LW, Chen DH, Huang JR, Pan K, Lin Y, Wu BT, Dai Y, et al: Integrated analysis of long non-coding RNAs and mRNA expression profiles reveals the potential role of lncRNAs in gastric cancer pathogenesis. Int J Oncol 45: 619-628, 2014.

21. Jia H, Osak M, Bogu GK, Stanton LW, Johnson R and Lipovich L: Genome-wide computational identification and manual annotation of human long noncoding RNA genes. RNA 16: 1478-1487, 2010.

22. Tafer H and Hofacker IL: RNAplex: A fast tool for RNA-RNA interaction search. Bioinformatics 24: 2657-2663, 2008.

23. Tafer H, Amman F, Eggenhofer F, Stadler PF and Hofacker IL: Fast accessibility-based prediction of RNA-RNA interactions. Bioinformatics 27: 1934-1940, 2011

24. Kontogeorgos $\mathrm{G}$ and Thodou E: The gonadotroph origin of null cell adenomas. Hormones (Athens) 15: 243-247, 2016.
25. Ishii Y, Suzuki M, Takekoshi S, Egashira N, Yamazaki M, Miyai S, Sanno N, Teramoto A and Osamura RY: Immunonegative 'null cell' adenomas and gonadotropin (Gn) subunit (SUs) immunopositive adenomas share frequent expression of multiple transcription factors. Endocr Pathol 17: 35-43, 2006.

26. Balogun JA, Monsalves E, Juraschka K, Parvez K, Kucharczyk W, Mete O, Gentili F and Zadeh G: Null cell adenomas of the pituitary gland: An institutional review of their clinical imaging and behavioral characteristics. Endocr Pathol 26: 63-70, 2015.

27. Zhu YP, Bian XJ, Ye DW, Yao XD, Zhang SL, Dai B, Zhang HL and Shen YJ: Long noncoding RNA expression signatures of bladder cancer revealed by microarray. Oncol Lett 7: 1197-1202, 2014.

28. Tremblay JJ, Lanctôt $\mathrm{C}$ and Drouin J: The pan-pituitary activator of transcription, Ptx1 (pituitary homeobox 1), acts in synergy with SF-1 and Pit1 and is an upstream regulator of the Lim-homeodomain gene Lim3/Lhx3. Mol Endocrinol 12: 428-441, 1998

29. Kolfschoten IGM, van Leeuwen B, Berns K, Mullenders J, Beijersbergen RL, Bernards R, Voorhoeve PM and Agami R: A genetic screen identifies PITX1 as a suppressor of RAS activity and tumorigenicity. Cell 121: 849-858, 2005.

30. Lamolet B, Pulichino AM, Lamonerie T, Gauthier Y, Brue T, Enjalbert A and Drouin J: A pituitary cell-restricted T box factor, Tpit, activates POMC transcription in cooperation with Pitx homeoproteins. Cell 104: 849-859, 2001.

31. Pellegrini-Bouiller I, Manrique C, Gunz G, Grino M, Zamora AJ, Figarella-Branger D, Grisoli F, Jaquet $\mathrm{P}$ and Enjalbert A: Expression of the members of the Ptx family of transcription factors in human pituitary adenomas. J Clin Endocrinol Metab 84: 2212-2220, 1999.

32. Wierinckx A, Auger C, Devauchelle P, Reynaud A, Chevallier P, Jan M, Perrin G, Fèvre-Montange M, Rey C, Figarella-Branger D, et al: A diagnostic marker set for invasion, proliferation, and aggressiveness of prolactin pituitary tumors. Endocr Relat Cancer 14: 887-900, 2007.

33. Qian ZR, Sano T, Yoshimoto K, Yamada S, Ishizuka A, Mizusawa N, Horiguchi H, Hirokawa M and Asa SL: Inactivation of RASSF1A tumor suppressor gene by aberrant promoter hypermethylation in human pituitary adenomas. Lab Invest 85: 464-473, 2005.

34. Pefani D-E, Latusek R, Pires I, Grawenda AM, Yee KS, Hamilton G, van der Weyden L, Esashi F, Hammond EM and O'Neill E: RASSF1A-LATS1 signalling stabilizes replication forks by restricting CDK2-mediated phosphorylation of BRCA2. Nat Cell Biol 16: 962-971, 1-8, 2014.

35. Huang KH, Huang SF, Chen I-H, Liao CT, Wang HM and Hsieh LL: Methylation of RASSF1A, RASSF2A, and HIN-1 is associated with poor outcome after radiotherapy, but not surgery, in oral squamous cell carcinoma. Clin Cancer Res 15: 4174-4180, 2009.

36. Krop I, Parker MT, Bloushtain-Qimron N, Porter D, Gelman R, Sasaki H, Maurer M, Terry MB, Parsons R and Polyak K: HIN-1, an inhibitor of cell growth, invasion, and AKT activation. Cancer Res 65: 9659-9669, 2005 .

37. Shigematsu H, Suzuki M, Takahashi T, Miyajima K, Toyooka S, Shivapurkar N, Tomlinson GE, Mastrangelo D, Pass HI, Brambilla E, et al: Aberrant methylation of HIN-1 (high in normal-1) is a frequent event in many human malignancies. Int $\mathrm{J}$ Cancer 113: 600-604, 2005.

38. Ladha J, Sinha S, Bhat V, Donakonda S and Rao SM: Identification of genomic targets of transcription factor AEBP1 and its role in survival of glioma cells. Mol Cancer Res 10: 1039-1051, 2012.

39. Zhou Y, Zhang X and Klibanski A: Genetic and epigenetic mutations of tumor suppressive genes in sporadic pituitary adenoma. Mol Cell Endocrinol 386: 16-33, 2014.

40. Sleiter N, Pang Y, Park C, Horton TH, Dong J, Thomas P and Levine JE: Progesterone receptor A (PRA) and PRB-independent effects of progesterone on gonadotropin-releasing hormone release. Endocrinology 150: 3833-3844, 2009. 